\title{
ORIGINAL ARTICLE Epigenetic regulation of the neuroblastoma genes, Arid3b and Mycn
}

\author{
K Kobayashi, LM Jakt and S-I Nishikawa
}

\begin{abstract}
AT-rich interaction domain molecule $3 B(A R I D 3 B)$ and $M Y C N$ are expressed in a portion of neuroblastoma, and form a combination that has strong oncogenic activity in mouse embryonic fibroblasts (MEFs). Here, we show that this combination can also convert neural stem cells to neuroblastoma-like tumor. To address whether there are common mechanisms regulating the expression of this combination of genes, we examined public repositories of gene expression data and found that although these genes are rarely expressed together, co-expression was observed in a proportion of germ cell tumors (GCTs), in embryonic stem (ES) cells and in testis. These cell types and tissues are related to pluripotency and we show here that in mouse ES cells, Arid3b and Mycn are indeed involved in cell proliferation; the former in avoiding cell death and the latter in driving cell cycle progression. Accordingly, the two genes are induced during somatic cell reprogramming to iPS, and this induction is accompanied by the switching of promoter histone marks from H3K27me3 to H3K4me3. Conversely, the switch from H3K4me3 to H3K27me3 in these genes occurs during the differentiation of neural crest to mature sympathetic ganglia cells. In many, if not most, neuroblastomas these genes carry H3K4me3 marks within their promoters. Thus, a failure of the epigenetic silencing of these genes during development may be an underlying factor responsible for neuroblastoma.
\end{abstract}

Oncogene (2013) 32, 2640-2648; doi:10.1038/onc.2012.285; published online 2 July 2012

Keywords: Arid3b; Mycn; neuroblastoma; embryonic stems cell; molecular network for pluripotency; epigenetics

\section{INTRODUCTION}

AT-rich interaction domain molecule $3 B(A R I D 3 B)$ is a member of a family of proteins that are characterized by an AT-rich interaction domain that was initially identified by its interaction with $R B 1$ in an acute myeloblastic leukemia cell line. ${ }^{1}$ The null mutant of Arid $3 b$ is embryonic lethal due to massive cell death of nascent neural crest. $^{2}$ As Mycn is implicated as a factor of cell cycle progression in neural crest cells, the phenotype of Arid $3 b(-/-)$ mice suggested that the combination of these two genes may also be involved in tumor formation. This idea was supported by our observation that a combination of $A R I D 3 B$ and MYCN is sufficient to confer tumorforming activity on mouse embryonic fibroblasts (MEFs) in immunodeficient mice. Moreover, $A R I D 3 B$ is expressed in most stage 4 neuroblastoma in which MYCN has been implicated in the oncogenic process. ${ }^{3}$ The effect of Arid3b expression in neural crest, neuroblastoma cell lines and MEFs, in concert, suggests that it has an ability to protect cells from apoptosis during Mycn-driven proliferation., 2 During mouse neural crest differentiation, concomitant expression of the two genes is essential for rapid generation of a sufficient number of cells, with Mycn promoting cell cycle progression and Arid $3 b$ preventing cell death. Conversely, the same set of genes, when expressed in an uncontrolled manner, should increase the danger of tumor transformation, and such expression may cause some proportion of neuroblastoma. Hence, expression of Arid $3 b$ needs to be strictly regulated during embryogenesis and in adult tissues to avoid inappropriate co-expression., ${ }^{2,4}$

Myc has been implicated as a major factor of the cell cycle in various cell and tumor types; the most intensely studied of which is embryonic stem (ES) cells. In mouse ES cells, it has been suggested that c-myc is involved in cell-autonomous growth through the regulation of the cell cycle, cell metabolism and chromosomal accessibility. ${ }^{5,6}$ Indeed, it has recently been proposed that Myc forms the core of an independent gene regulatory module that has an essential role in the maintenance of self-renewal and pluripotency of ES cells, together with a core pluripotency and a polycomb module. ${ }^{7}$ One problem of using Myc as a factor of cell cycle progression is its ability to induce apoptosis. In tumor cells, it is known that a high level of $M y c$ induces cell death by both p53-dependent and -independent means. ${ }^{8,9}$ Hence, transformation of cells by Myc requires additional mechanism(s) to inhibit cell death. Mutations of genes in the p53Arf pathway, overexpression of anti-apoptotic molecules such as bcl2 and mutations in bim 1 have been implicated in apoptosis avoidance in tumors. ${ }^{10}$ In ES cells, however, the molecular mechanism underlying cell protection from Myc-induced apoptosis remains unknown.

In this study, we attempted to reveal common mechanisms underlying the concomitant expression of Arid3b and Mycn. We show here, that both genes are expressed in pluripotent cells such as mouse ES and iPS cells, and have a specific role in the proliferation of ES cells. Our result also demonstrate that their expression is associated with the program of pluripotency, as their epigenetically silenced expression is derepressed only at the final stage of iPS reprogramming and is accompanied by a switch of promoter histone marks from H3K27me3 to H3K4me3. Conversely, the two genes are epigenetically silenced upon maturation of sympathetic ganglia cells through the switch from H3K4me3 to H3K27me3. As the chromatin of the two genes is found in an activated state in neuroblastoma, this raises the possibility that a 
failure in switching from active to repressed chromatin underlies neuroblastoma generation.

\section{RESULTS}

Overexpression of Arid $3 b$ and Mycn transforms neural stem cells (NSCs) to neuronal tumor

We previously showed that overexpression of Arid3b and Mycn convert MEFs to tumor cells. ${ }^{3}$ In this process, Mycn promotes cell cycle progression whereas Arid $3 b$ inhibits apoptosis. To investigate whether or not this combination has the same effect on neural cells, we used a lentiviral vector to transduce the two genes into NSCs (Figure 1a). The resulting cells were cultured for a further 2 weeks under NSC maintenance conditions, characterized and finally injected into nu/nu mice to test their tumorigenicity. Overexpression of Mycn in NSCs enhanced cell growth in vitro, but, like in MEFs, induced apoptosis as detected by the proportion of Annexin ${ }^{+} / 7-\mathrm{AAD}^{-}$cells. Arid3b itself did not drive cell growth, but showed an ability to suppress Mycn-induced apoptosis.
Consequently, cell growth is markedly enhanced by transduction of both genes together (Figures $1 \mathrm{~b}$ and $\mathrm{c}$ ). In nude mice, the cells transduced with Mycn alone as well as Arid $3 b+M y c n$ together formed tumors, whereas those transduced with Arid3b alone did not. However, cells transduced with both genes developed much more aggressive tumor than Mycn alone (Figure 1d).

Both induced tumors developed a dense proliferation of small round cells with prominent and large nucleoli reflecting a clinical hallmark of MYC amplified tumors. Among those, tumor induced by Mycn alone was characterized by a large degree of apoptosis. As the histological picture lacked morphological evidence of differentiation toward specific lineages, we classified it as undifferentiated sarcoma according to the pathological criteria. In contrast, tumor formed from cells co-transducted with Arid3b and Mycn exhibited less apoptosis and occasionally contained rosette-like structures with clear neurophil formation. Their characteristic pathological features, and expression of differentiated peripheral neuronal markers such as tyrosine hydroxylase and synaptophysin are collectively consistent with a pathological a

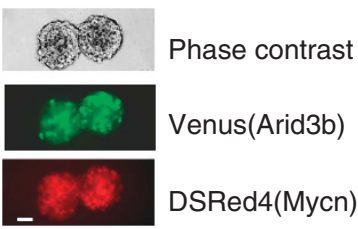

b $\left(\times 10^{4}\right)$

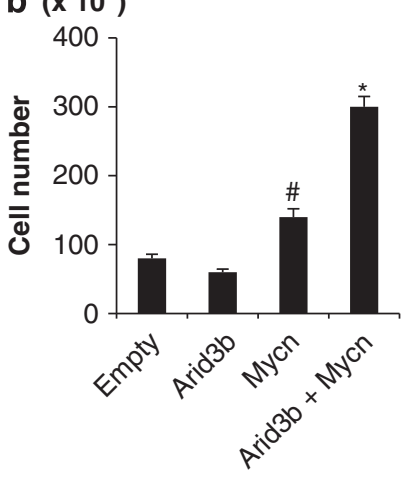

e
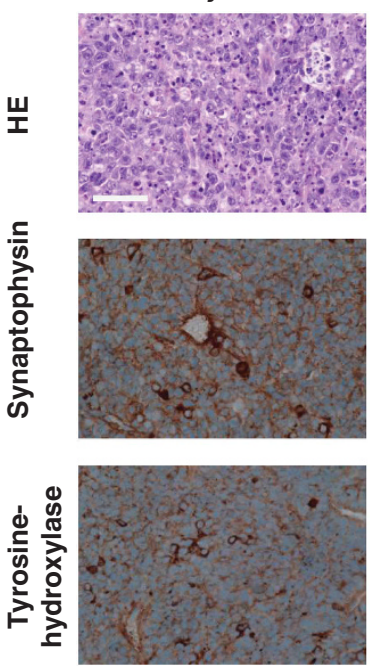

C

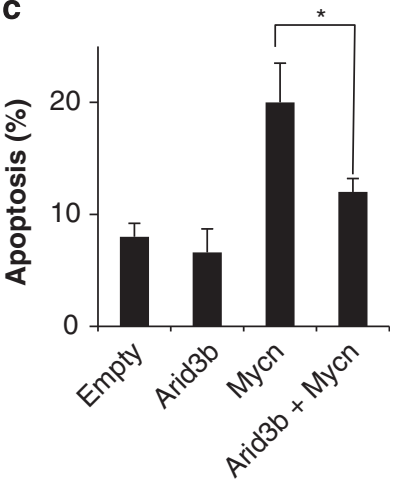

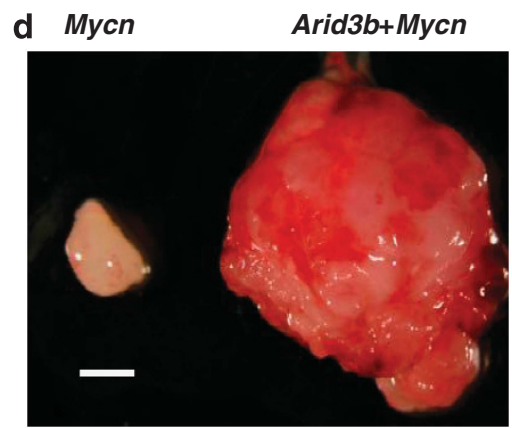

Figure 1. Overexpression of Arid3b and Mycn transforms NSCs to neuronal tumor. (a) NSCs grown in maintenance conditions as neurospheres. Co-transfection of both Arid $3 b$ and Mycn was indicated by the co-expression of each reporter protein in the same cells. Scale bar, $50 \mu \mathrm{m}$. (b) Effect of Arid3b, Mycn or Arid3b + Mycn on the proliferation of NSCs. Mycn drives cell growth in culture; furthermore co-transfection of Arid3b on Mycn-overexpressing cells further enhanced cell growth. Error bars represent s.d. from three independent replicates. $P$-values were calculated against the control (empty) using a two-tailed Student's $t$-test. ${ }^{*} P<0.05$ and ${ }^{*} P<0.01$. (c) Cell apoptosis (Annexin ${ }^{+}$and 7-AAD ${ }^{-}$) was quantified by flow cytometry. Arid3b suppressed apoptosis induced by Mycn. Error bars represent s.d. from three independent replicates. $P$-value was calculated between Mycn and Arid $3 b+$ Mycn transduced cells using a two-tailed Student's $t$-test. ${ }^{*} P<0.01$. (d) Gross appearance of representative tumors, transduced by Arid $3 b+$ Mycn (right) and by Mycn alone (left). Scale bar, $5 \mathrm{~mm}$. Transduced NSCs were subcutaneously injected into BALB/c nu/nu mice and generation of tumors was monitored by weekly inspection of the injected site. Six weeks after the injection, mice were killed for pathological analysis of the tumors. (e) Hematoxylin-eosin and immunohistochemical analysis of the induced tumors; Arid $3 b+$ Mycn (right) and by Mycn alone (left). Scale bars, $50 \mu \mathrm{m}$. 
classification of (Arid3b + Mycn-induced tumor) neuroblastoma according to the criteria established by the International Neuroblastoma pathology Classification. ${ }^{11}$

Taken together, as is the case for MEFs, the combination of Arid3b and Mycn can induce transplantable tumor in neural progenitor cells, with Mycn acting to promote cell cycle progression and Arid $3 b$ inhibiting apoptosis.

Microarray analysis of Arid3b and Mycn expression in tumor cells and pluripotent cell lines

Our observation that overexpression of Arid3b and Mycn together is sufficient to transform NSCs and MEFs to tumor cells prompted us to search for other malignancies expressing this combination in public repositories of microarray data (GEO and ArrayExpress). We examined expression of these genes in a total of 2666 samples representing 2064 tumor samples ( $\sim 24$ tumor types) and 602 non-tumor samples (tissues and cell lines). Within the 24 tumor types examined, enrichment of $A R I D 3 B$ expression was observed in only 4 types of tumor; acute lymphoblastic leukemia, acute myeloblastic leukemia, GCT and neuroblastoma; whereas MYCN expression was observed in 7 types of tumors. Significant coexpression of $A R I D 3 B$ and MYCN was observed in only acute myeloblastic leukemia, GCT and neuroblastoma (Supplementary Table 2). Co-expression of Arid3b and Mycn was also observed in a small number of non-tumor samples: ES cells, embryoid bodies and fetal (but not adult) hematopoietic stem cells and in testis tissue. Both ESCs and fetal hematopoietic stem cells are cells that undergo expansion and is consistent with our idea that coexpression of these two genes only occurs in expanding populations.

We were intrigued by the observation that the two genes are expressed in three cell types that express both Oct4 and Nanog, a gene set for pluripotency. ${ }^{5-7}$ We thus decided to further investigate the significance of this observation in ES cells.

To confirm the expression of Arid $3 b$ and Mycn in ES cells, we measured the expression of Arid3b, c-myc and Mycn by quantitative reverse transcription-PCR in ES cells either cultured in a standard LIF-containing condition or in a defined medium containing inhibitors of extracellular signal-regulated kinase and glycogen-synthase kinase-3B (2i) that maintain the pluripotency of ES cells in the absence of exogenous growth factors (considered as the ground state of pluripotency). ${ }^{12}$ The expression level of Nanog was also measured as an ES cell specific control. All genes (Mycn, Arid3b, Nanog and c-myc) are expressed in ES cells cultured in standard LIF-containing conditions. However, c-myc expression was reduced significantly in ES cells at the ground state (2i), whereas other genes remain unchanged (Figure 2).

\section{Arid3b protects ES cells from Mycn-induced apoptosis}

Although the molecular network for pluripotency (P-NW) in ES cells with Oct4/Sox2/Nanog at its core has been characterized to a great extent, ${ }^{5-7}$ which genes are involved in the proliferation of pluripotent cells is not clear. Prompted by the expression of Arid3b and Mycn in ES cells, we considered the possibility that these genes support the proliferation of ES cells. As Arid3b (-/ - ) ES cells proliferate normally in LIF-containing standard culture conditions, Arid $3 b$ should be dispensable for ES cell proliferation. However, it is likely that multiple mechanisms are involved in ES cell proliferation. To assay the effect of Arid3b at higher sensitivity, we generated an Arid3b (-/ ) ES cell line in which Flag-tagged Arid $3 b$ is expressed under the control of doxycycline (dox) and examined the proliferation of ES cells under $2 \mathrm{i}$ conditions with or

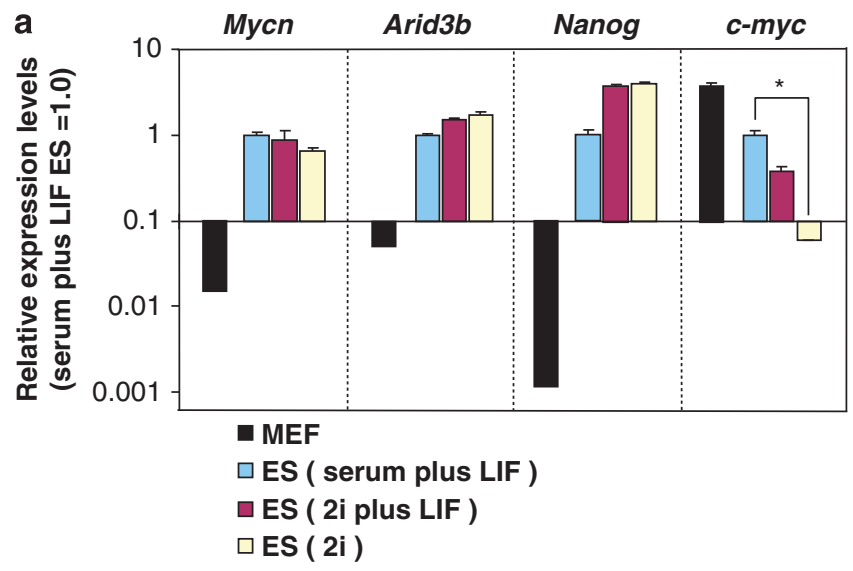

b
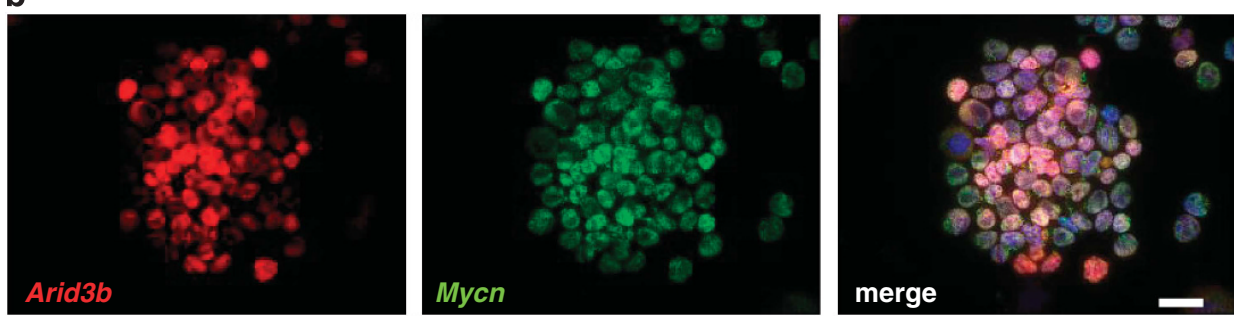

Figure 2. Expression of Arid3b and Mycn in ES cells. (a) Quantative reverse transcription-PCR measurement of Mycn, Arid3b, Nanog and c-myc in MEF and ES cells. ES cells were maintained in different culture conditions: serum plus LIF, 2i or 2i plus LIF. MEF were maintained in serum plus LIF conditions as a negative control. Each graph shows relative expression levels compared with that in ES cells (serum plus LIF ES $=1.0$ ). Error bars represent s.d. from three independent replicates. Statistical analysis in c-myc expression level was calculated between the serum plus LIF and $2 \mathrm{i}$ condition using a two-tailed Student's $t$-test. ${ }^{*} P<0.01$. (b) Arid $3 b$ and Mycn co-localize in ES cells grown in $2 \mathrm{i}$ condition. Panel shows indirect immuno-fluorescent staining with the indicated antibodies. Nuclei were stained with $4^{\prime} 6$-diamidino-2-phenyl indole. Scale bars, $20 \mu \mathrm{m}$. 
without Arid3b. Repression of Arid3b showed little effect on ES cell growth under standard conditions (serum plus LIF). However, when maintained in the absence of exogenous growth factors (2i), ES cells grow slower in the absence of Arid3b (Figure 3a) and this growth deficit becomes more overt at low cell densities (Figure 3b). In contrast, expression levels of the pluripotent markers such as Oct4/ Sox2/ Nanog were largely unaffected (data not shown), suggesting that $A r i d 3 b$ is not directly involved in the maintenance of pluripotency.

To investigate whether or not the action of Arid3b and Mycn in ES cells is similar to that in MEFs and neural cells, we performed cell cycle analysis of ES cells before and after repression of transgenic Arid3b by dox in the absence or presence of siRNAs inhibiting Mycn. Flow cytometric analyses of both cell cycle and apoptosis revealed that repression of Arid $3 b$ in the presence of Mycn ( - siRNA) caused no significant effect on the extent of cell cycle progression, but did result in a significant increase in the number of Annexin ${ }^{+} / 7-A^{-} D^{-}$cells indicating an induction of apoptosis. This increase of apoptosis was suppressed by Mycn knock down. Hence, at least one role of Arid3b in ES cells is to protect cells from apoptosis. Interestingly, cell cycle progression was inhibited only when both Arid $3 b$ and Mycn were simultaneously suppressed, suggesting a latent and redundant Arid $3 b$ function in driving proliferation in ES cells in the absence of both
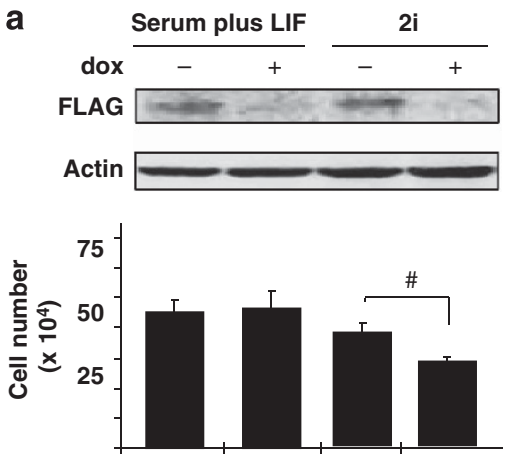

Plating cell number $\left(10 \times 10^{4} / 10 \mathrm{~cm}^{2}\right)$

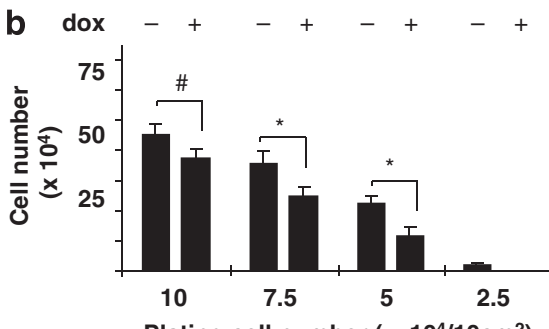

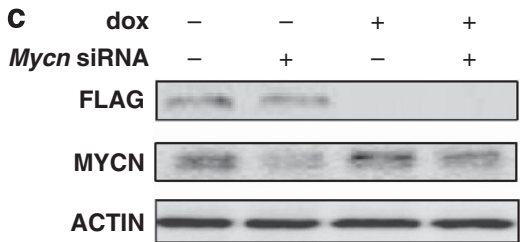
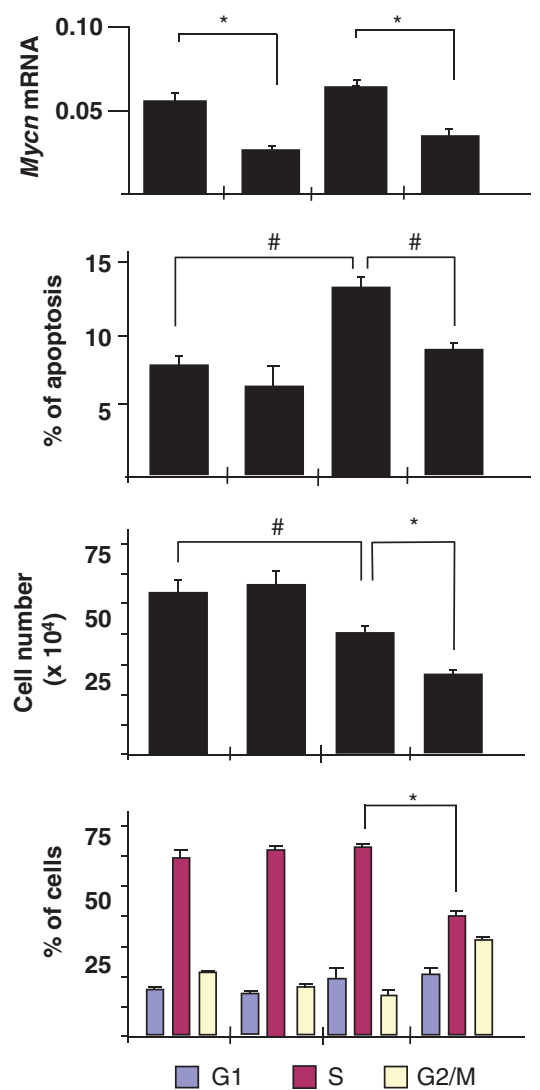

Figure 3. Arid $3 b$ protects ES cells from Mycn-induced apoptosis. Arid3b null ES cells carrying a construct containing FLAG-tagged Arid $3 b$ driven by a dox-repressable promoter were established and used to analyze the effect of Arid3b expression in ES cells. Error bars represent s.d. from three independent replicates. Statistical significance was calculated by Student's $t$ test. ${ }^{*} P<0.05$; ${ }^{*} P<0.01$. (a) Dox-induced repression of the Arid3b-transgene in ES culture conditions. Western blots of whole-cell extracts using antibodies against FLAG (Arid3b) and $\beta$-actin (loading control). Detection of flag-tagged Arid3b protein is lost after 2 days exposure of dox (upper panel). The proliferation of the same ES cell line was compared with or without repression of Arid $3 b$ under either serum plus LIF or $2 \mathrm{i}$ conditions. ES cells were plated at a density of $10^{4}$ cells/ $\mathrm{cm}^{2}$, and the total cell number determined after 2 days (lower panel). (b) Effect of cell density on the proliferation of ES cells. Cell growth was evaluated (as in a) at different starting cell densities under $2 \mathrm{i}$ conditions. (c) Function of Arid3b and Mycn in ES cell proliferation under $2 \mathrm{i}$ conditions. The expression of Arid $3 b$ and Mycn were repressed by dox or siRNA, respectively. All assays were performed 2 days after transfection and or application of dox. Western blots using antibodies against FLAG (Arid3b), Mycn and $\beta$-actin (loading control) shows each depleted level (top). Mycn mRNA levels were also determined by quantative reverse transcription-PCR analysis relative to ubiquitin levels (second panel). The proportion of apoptotic cells was determined by flow cytometric analysis (third panel). Annexin ${ }^{+}$and $^{7-A A D}{ }^{-}$cells were considered to be apoptotic. Cell numbers were determined (fourth panel) and analyzed for bromodeoxyuridine incorporation by flow cytometry to determine the proportions of cells at different stages of the cycle (bottom panel). 
c-myc and Mycn (Figure 3c). These results are consistent with our previous observations of MEF, neural cells and neuroblastoma in that Mycn is the primary factor of cell cycle progression whereas Arid $3 b$ protects the cells from apoptosis.

Induction of Arid $3 b$ and Mycn during iPS reprogramming

Pluripotency can be induced in somatic cells by the overexpression of a small number of transcription factors. ${ }^{13}$ However, such reprogramming, in addition to being inefficient (in that small numbers of ES-like colonies are formed), usually results in the generation of both partially reprogrammed (piPS) and fully reprogrammed (iPS) cells. ${ }^{14-16}$ ES, iPS and piPS are indistinguishable in terms of proliferation and morphology. However, piPS cells maintain the expression of the exogenous reprogramming factors and generally fail to activate a number of pluripotency-associated factors (including Nanog and Dnmt3/). Therefore, piPS cells can be considered to have many of the properties of ES cells without having activated the P-NW. ${ }^{14-16}$ To test whether expression of Arid3b and Mycn is directly downstream of exogenously transduced reprogramming factors (that is, Oct4, Sox2, KIf4 and c-myc) or an outcome of the implementation of the P-NW by reprogramming, we generated both iPS and partially reprogrammed iPS (piPS) cells from NSCs and assessed the expression of Arid3b and Mycn by quantitative reverse transcription-PCR. The piPS cells analyzed here correspond to cells obtained at the intermediate stage of reprogramming after the transduction of the reprogramming factors. ${ }^{14-16}$ As previously reported, ES, iPS and piPS cells were indistinguishable from each other in terms of proliferation and morphology; however, expression of the exogenously transduced factors persisted in the piPS cells and we detected expression of Nanog expression only in ES and iPS cells (Supplementary Figure 1A-D). Expression of Arid3b and Mycn were detectable in fully reprogrammed iPS at a comparable level to that of ES cells, but only at low levels in piPS (Figure 4a). We also confirmed these results in a previously published DNA microarray data set (Supplementary Figure 1E). ${ }^{16}$

We next investigated whether or not the expression of the two genes is accompanied by the reprogramming of epigenetic marks. The UCSC genome Bioinformatics data base that contains an extensive collection of chromatin immunoprecipitation data, indicated that the promoters of the two genes are occupied by H3K4me3 (active) but not H3K27me3 (inactive) marks in ES cells, whereas the opposite pattern was found in MEF (Supplementary Figure 1F). To extend these observations, we performed chromatin immunoprecipitation analysis to determine the histone marks present in the promoters of these genes in several cell types including NSC, piPS and iPS. We also analyzed the methylation of CpG islands overlapping with the promoters and found that the methylation pattern is mostly unchanged; being hypomethylated in NSCs and throughout the reprogramming process (Figure 4b). On the other hand, both genes are marked by repressive H3K27me3 in piPS cells and NSCs, and are only marked by H3K4me3 in iPS and ES cells (Figure 4c). Thus, expression of the two genes during iPS formation is accompanied by an epigenetic reprogramming of histone marks but not methylation of CpG islands.

Misregulation of histone modification in neuroblastoma at Arid3b and Mycn promoters

The change in histone modification at the promoters of Arid $3 b$ and Mycn from a closed to an open state during reprogramming of NSCs prompted us to investigate the histone modification at these genes during the development of normal sympathetic neurons. Neuroblastoma is derived from neural crest and shares many common features with developing neural ganglia such as expression of Mycn. ${ }^{17,18}$ To define the difference between a
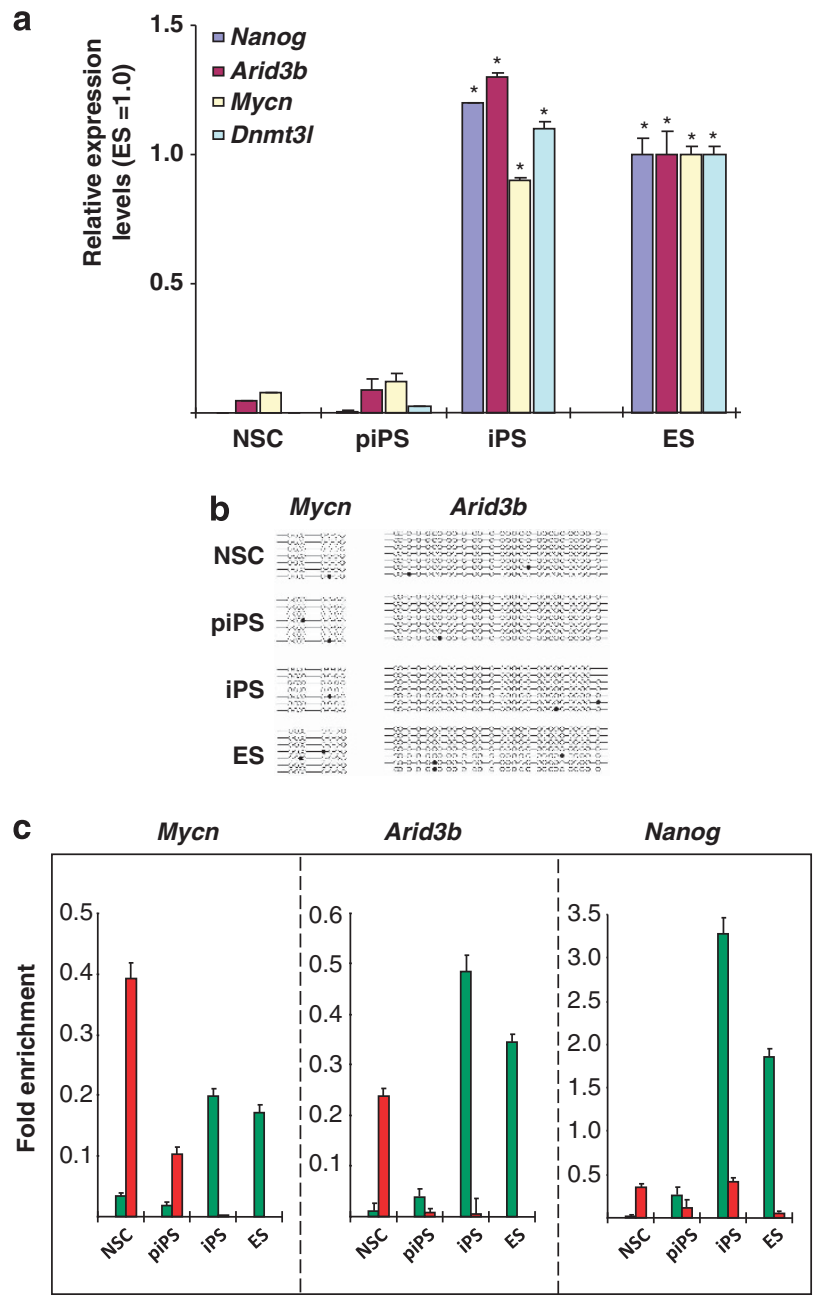

पH3K4me3 H3K27me3

Figure 4. Induction of Arid $3 b$ and Mycn during iPS reprogramming (a) Quantative reverse transcription-PCR measurements of Nanog, Dnmt3l, Arid3b and Mycn expression in NSCs, pre-iPS (piPS) and iPS cells. Each graph shows relative expression levels compared with that in ES cells (1.0). Error bars are s.d.s. $P$-value was calculated against NSC expression using a two-tailed Student's $t$-test. $* P<0.01$. (b) Methylation status of Arid3b and Mycn CpG islands. Each horizontal line indicates a single clone from the respective PCR products after bisulfite treatment. Open circles indicate lack of methylation and filled circles represent methylated cytosine. (c) Chromatin immunoprecipitation analysis for H3K4me3 and H3K27me3 at Mycn, Arid3b and Nanog promoters. Data are represented as fold enrichment of precipitated DNA associated with a given histone modification relative to input chromatin. Active or non-repressed histone modifications (H3K4me3, green) were only observed in iPS and ES cells. Error bars represent s.d.s. Detection level from pull down from normal mouse immunoglobulin $\mathrm{G}$ levels are negligible and data not shown.

neuroblastoma and normal cells, we assessed the expression of the two genes during mouse sympathetic cervical ganglia (SCG) development. At embryonic day 11.5 (E11.5 or 11.5 days post-coitus) both Arid3b and Mycn expression was detected in the SCG. Arid3b expression was also detected in the notochord, dorsal root ganglia and developing progenitors of adrenal medulla (Figures $5 \mathrm{a}$ and b). Quantitative reverse transcription-PCR measurements indicated that the expression of both genes is gradually downregulated during SCG development after E11.5 and their expression is lost completely in the adult SCG. Thus, the 
two genes are repressed along with SCG maturation. Consistent with the decrease of expression, the histone marks at the respective gene promoters shifted from H3K4me3 to H3K27me3 (Figure 5b). The change to repressive H3K27me3 marks was complete at the Mycn locus, whereas some H3K4me3 modification was still detected at the Arid3b promoter in adult SCG.
Nonetheless, both genes contained mostly H3K27me3 marks in adult SCG.

We next analyzed the histone modifications at these genes in a mouse neuroblastoma cell line (C-1300). In C-1300, the two genes are expressed, albeit at a low level. However, only H3K4me3 was detected at the promoters of the two genes. This implies that a a

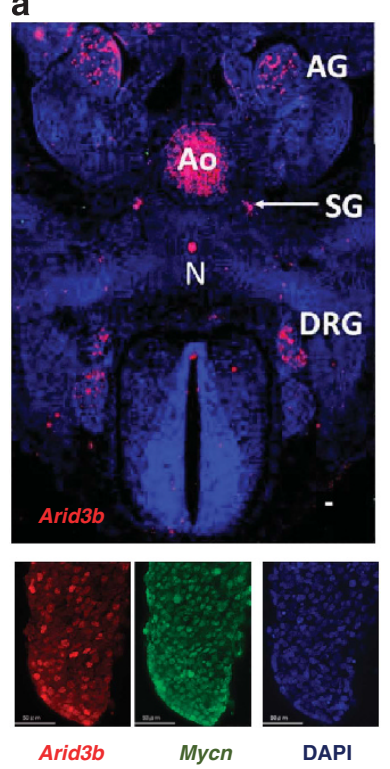

b
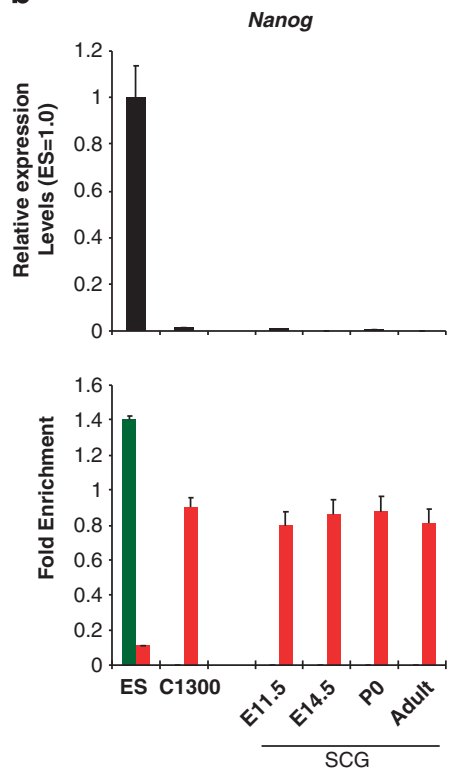
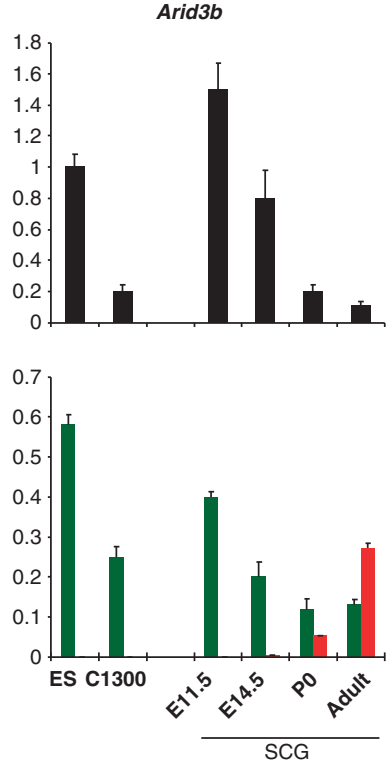

\section{(1)}

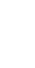

(n) 
failure in the shift of histone marks from H3K4me3 to H3K27me3 at these genes is a characteristics of neuroblastoma. As we could obtain only one mouse neuroblastoma cell line, we next analyzed the histone modifications of these genes in six human cell lines; keratinocyte-derived iPS cells, keratinocytes and four neuroblastomas with or without MYCN amplification. Neither gene was detected in keratinocytes; in contrast, a low but significant expression of $A R I D 3 B$ was detected in all neuroblastomas, irrespective of the presence of $M Y C N$ amplification, whereas high $M Y C N$ expression was found only in the line bearing MYCN amplification. Irrespective of the expression level, the active $\mathrm{H} 3 \mathrm{~K} 4 \mathrm{me} 3$, but not the repressed H3K27me3 mark was enriched at the $A R I D 3 B$ promoter in all neuroblastoma cell lines. A similar pattern was also observed on the promoter of $M Y C N$, except in the one cell line (Figure $5 \mathrm{c}$ ). Although a relatively good correlation of the H3K27me3 mark and gene repression was observed, the expression level of these genes in neuroblastoma cell lines varied significantly.

\section{DISCUSSION}

The combination of Arid3b and Mycn expression has a strong oncogenic activity in MEFs. ${ }^{3}$ Here, we show that NSCs transduced with this gene combination are capable of generating neural tumor that can grow in subcutaneous tissue of nu/nu mice. Unlike MEFs that require both Arid3b and Mycn for transformation, Mycn alone is sufficient to transform NSCs to tumor. However, NSCs transduced with both genes resulted in more malignant tumor, presumably due to the repression of apoptosis by Arid3b (Figure 1c). Thus, in concert, our observations demonstrate that the role of Arid3b in development and tumor formation is to suppress apoptosis in cells undergoing Mycndependent proliferation.

By data-mining repositories of expression data of tumors and normal cell lines, we found that tumors expressing the $A R I D 3 B$ and MYCN combination are unexpectedly rare; co-expression was only enriched in three tumor types (neuroblastoma, acute myeloblastic leukemia and male GCT). Co-expression of the two genes was also detected in a small number of non-tumor samples. Of the 26 (non-tumor) positive samples that we identified (from 602 samples), 11 were ES or ES-derived embryoid body cells, 6 were testicular samples and 9 were hematopoietic stem cells (mostly fetal, co-expression is not usually observed in adult hematopoietic stem cells).

Of these, we were intrigued by the result that co-expression is found in male GCT, testicular tissue and ES cells, all of which can be considered as having some features of pluripotency. This raises the possibility that the expression of Arid $3 b$ and Mycn are implemented in the molecular network of pluripotency (P-NW) ${ }^{5,19}$ In this study, we used the 2i-defined conditions for ES cell culture, as this condition, because of the absence of exogenous growth factors, illuminates the intrinsic P-NW more clearly. ${ }^{12}$ Under this condition, the only Myc family member expressed is Mycn. This is consistent with previous work indicating a LIF-dependent induction of c-myc. ${ }^{20-22}$ Expression of Arid3b and Mycn in ES cells cultured in $2 \mathrm{i}$ medium implies that these genes are integrated with the P-NW. Consistent with this observation are recent studies showing that the two molecules are indeed included in the molecular network for pluripotency. ${ }^{5,19}$ That Arid3b and Mycn is integrated with P-NW is also supported by our findings that the two genes are reactivated during reprogramming from NSC to iPS. This reactivation of expression does not occur in partially reprogrammed cells (piPS) and is accompanied by a switch from repressive H3K27me3 to permissive H3K4me3 histone marks. These observations prompted us to consider the possibility that aberrant epigenetic regulation of the embryonic growth program can result in the formation of embryonic tumors, such as neuroblastoma.
It is widely accepted that a group of child cancers arise in embryonic cells that fail to shift from embryonic to mature states. ${ }^{23}$ Although definite genomic lesions such as anaplastic lymphoma kinase mutation and MYCN gene amplification have been identified in some neuroblastoma subtypes, molecular mechanisms that can account for the frequent incidence of spontaneous regression remain unidentified. ${ }^{23,24}$ As spontaneous regression generally leads to complete recovery from disease, such tumors may be free from major genetic alterations. Hence, epigenetic mechanisms are suspected to have a role in neuroblastoma and other tumor types that often undergo spontaneous regression. Interestingly, we found the presence of active H3K4me3 marks at the promoters of $A R I D 3 B$ and $M Y C N$ in human neuroblastoma lines. In contrast, we found that $\mathrm{H} 3 \mathrm{~K} 27 \mathrm{me} 3$ is found at the promoters of these genes in most normal cell types including NSCs. The expression level of the two genes might be influenced by other factors such as molecular networks in each neuroblastoma; however, it is possible that a failure of epigenetic silencing underlies the development of neuroblastoma. Silencing at these genes does indeed take place during normal development of SCG neurons from neural crest, and the switch from active to repressive histone marks on the promoters underlies this. It is thus likely that a failure of epigenetic repression in cells during SCG development would result in sustained expression of Arid3b and Mycn leading to inappropriate proliferation and tumor formation. As the overexpression of the two genes can convert NSCs to tumor inducing cells, it is conceivable that sustained expression of the two genes can cause neuroblastoma. Taken together, we would suggest that some neuroblatomas arise as a result of a failure in epigenetic silencing.

A similar possibility has been suggested for Wilms' tumor; the most common pediatric kidney cancer. ${ }^{25}$ It was shown that: (1) many genes involved in renal development and expressed in renal stem cells carry active H3K4me3 marks at their promoters, (2) the factors and signal pathways involved in Wilms' tumor are largely distinct from those defined in adult cancers. It was also suggested that the overall chromatin structure of Wilms' tumor cells is reminiscent to that of ES cells. ${ }^{25}$ These observations, thus implicate that epigenetic failure in gene silencing during development is rather common cause of pediatric tumors that arise during embryonic development.

\section{MATERIALS AND METHODS}

Cells and culture condition

Mouse ES and iPS cells were cultured on gelatin-coated dishes in (a) serum plus $\mathrm{LIF}^{26}$ or (b) a defined serum-free medium consisting of N2B27 medium in the presence two inhibitors, CHIR99021 and PD0325901 (2icondition). ${ }^{12}$ For cell proliferation assays, single cell suspensions were prepared using trypsin-EDTA and cells were seeded at $10^{4} \mathrm{cell} / \mathrm{cm}^{2}$, or as specified. (c) Mouse NSCs were established and maintained as previously described. ${ }^{27}$ (d) Human neuroblastoma cells (SH-SY5Y, NB-69, IMR-32 and CHP-126) and the mouse neuroblastoma cell (C-1300) were cultured as described. ${ }^{3}$ (e) Human iPS cell induction from adult skin keratinocytes was performed as in Ban et al. ${ }^{28}$ (f) SCG were dissected from mouse embryos, as described. ${ }^{29}$ Collected cells were directly used for subsequent chromatin immunoprecipitation and mRNA analysis.

Generation of fully reprogrammed and partially reprogrammed mouse iPS cells

Several mouse iPS cells used in this study have been previously described. $^{30}$ To generate novel mouse iPS and piPS cells, NSCs isolated from Oct-4/green fluorescent protein transgenic mice ${ }^{31}$ were used. The protocols to generate iPS and piPS cells are illustrated in Supplementary Figure 1A. piPS cells generated in this study can be converted to fully reprogrammed iPS by culture in $2 \mathrm{i}$ conditions (data not shown) as reported. ${ }^{14,15}$ 
Gene transfection by lentiviral vector and tumorigenicity assay The lentiviral expression vectors, CS2-CMV-RfA-IRES-Venus and CS2-CMVRfA-IRES-DsREDT4 were used, respectively, to overexpress Arid3b and Mycn. Production and infection of lentiviral vectors were performed as described previously. ${ }^{32}$ NSCs were infected with a lentivirus carrying Arid3b, Mycn or both. Control cells were infected with CS2-CMV-RfA-IRES-Venus (empty). Following purification of Venus + or DsREdT4 ${ }^{+}$cells by FACS, $10^{6}$ NSCs

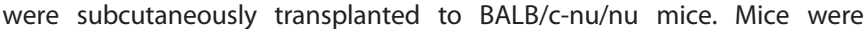
monitored weekly and the tumor diameter was measured with precision callipers. All animals used in this study were treated in accordance with the regulations on Animal Experimentation of the RIKEN Center for Developmental Biology.

\section{Immunohistochemistry}

Four-micrometer sections of tumor samples were stained with hematoxylin and eosin and adjacent sections were used for immunohistochemical analysis. Primary antibodies (used at a 1:100 dilution) were antisynaptophysin (clone SY38: Chemicon, Temecula, CA, USA) and tyrosine hydroxylase (clone IB5: Novocastra, Newcastle upon Tyne, UK). The BenchMark XT immunostainer (Ventana Medical Systems, Tucson, AZ, USA) and 3-30 diaminobenzidine detection kits (Ventana Medical Systems) were used for visualization. ES cells grown in cultures were stained with primary antibodies of Arid3b (ab32481: Abcam, Cambridge, MA USA), Mycn (ab16898: Abcam), respectively. Cells fixed with 4\% para-formaldehyde for $30 \mathrm{~min}$ were briefly permeabilized with $0.2 \%$ Triton X-100. Dishes were incubated with the primary antibody for $1 \mathrm{~h}$ followed by incubation with an appropriate secondary antibody for an additional hour. Nuclear staining with 4'6-diamidino-2-phenyl indole was performed after double labeling had completed. Mouse embryo were perfused with $4 \%$ para-formaldehyde and postfixed for $2 \mathrm{~h}$ before embedding in optimal cutting temperature compound (OCT). Eight-micrometer sections were processed and stained as described above.

\section{Arid3b inducible null ES system}

Flag-tagged Arid $3 b$ was inserted into the piggyBac (PB)-based expression cassette under control of a dox-responsive promoter (plasmids generated by Guo et al. ${ }^{33}$ and modified by $\mathrm{H}$ Niwa). Dox-responsive promoter plasmids were modified to monitor transgene expression by IRES-linked CD90.1 surface staining. These plasmids were co-transfected with PB-CAGtTA-IRESNeo and PBase expression plasmids (gifts from $\mathrm{H}$ Niwa and Guo et al.) using X-fect (Cat. no. 631317: Clontech, Clontech Mountain View, CA, USA) in Arid3b null ES cells, ${ }^{2}$ followed by the isolation of dox-responsive stable lines. The transgene expression was suppressed by adding $0.01 \mu \mathrm{g} /$ $\mathrm{ml}$ dox (Cat. no. 631311: Clontech) and in turn induced by dox withdrawal.

\section{Flow cytometry}

For cell cycle analyses, ES cells were pulse labeled with $10 \mu \mathrm{M}$ bromodeoxyuridine for $30 \mathrm{~min}$. Bromodeoxyuridine incorporation assays were performed using the bromodeoxyuridine FITC Flow Cytometry kit (BD Pharmingen, San Diego, CA, USA). Apoptosis was examined by Annexin-V and 7-Actinomycin $D$ (7-AAD) double staining (BD Pharmingen) following the manufacturer's protocol.

\section{Bisulfite genomic sequencing}

Genomic DNA was isolated using the DNeasy Blood and Tissue kit (Cat. no. 69506: Qiagen, Valencia, CA, USA) and converted with the EpiTect Bisulfite Kit (Cat. no. 59104: Qiagen). CpG regions were amplified with primers (listed in Supplementary Table 1). PCR products were sub-cloned into pCR2.1 vector using the TOPO TA Cloning Kit (Cat. no. K4500-01: Invitrogen, Carlsbad, CA, USA) and sequenced.

\section{Histone modification analysis}

Chromatin immunoprecipitation analysis was performed as described previously. ${ }^{34}$ In brief, chromatin fractions were treated with micrococcal nuclease (2910A: Takara, Kyoto, Japan) and immunoprecipitation was performed with Dynabeads (DB11202: Dynal Biotech ASA, Oslo, Norway). Antibodies used were as follows: anti-H3K4me3 (MABI0304: Wako Pure Chemical Industries Ltd, Osaka, Japan), H3K27me3 (6002: Abcam), normal mouse immunoglobulin G (SC-2025: Santa Cruz Biotechnology, Santa Cruz, CA, USA). Eluates were analyzed by quantitative PCR.
Reverse transcription-PCR, qPCR and gene expression analysis Total RNA was isolated using the RNeasy Plus mini kit (Qiagen) and reverse transcribed using Superscript III First strand Synthesis Supermix (Invitrogen). Real-time quantitative PCR was performed using SYBR Green detection as described. ${ }^{3}$ Primer sequences used in expression analysis, bisulfite sequencing and histone modification analysis are listed in Supplementary Table 1.

Protein extracts were processed for sodium dodecyl sulfatepolyacrylamide gel electrophoresis according to standard methods. Primary antibodies: FLAG (F3165: Sigma, St Louis, MO, USA), $\beta$-actin (A5316: Sigma). Secondary antibodies were as follows: anti-mouse immunoglobulin $\mathrm{G}(\mathrm{H}+\mathrm{L})$ horseradish peroxidase conjugate (81-6520: Zymed Laboratories, South San Francisco, CA, USA) and anti-rabbit immunoglobulin G, horseradish peroxidase-linked antibody (7047: Cell Signaling Technologies, Danvers, MA, USA).

\section{CONFLICT OF INTEREST}

The authors declare no conflict of interest.

\section{REFERENCES}

1 Numata S, Claudio PP, Dean C, Giordano A, Croce CM. Bdp a new member of a family of DNA-binding proteins, associates with the retinoblastoma gene product. Cancer Res 1999; 59: 3741-3747.

2 Takebe A, Era T, Okada M, Martin Jakt L, Kuroda Y, Nishikawa S. Microarray analysis of PDGFR alpha + populations in ES cell differentiation culture identifies genes involved in differentiation of mesoderm and mesenchyme including ARID3b that is essential for development of embryonic mesenchymal cells. Dev Biol 2006; 293: 25-37.

3 Kobayashi K, Era T, Takebe A, Jakt LM, Nishikawa S. ARID3B induces malignant transformation of mouse embryonic fibroblasts and is strongly associated with malignant neuroblastoma. Cancer Res 2006; 66: 8331-8336.

4 Sawai S, Shimono A, Wakamatsu Y, Palmes C, Hanaoka K, Kondoh H. Defects of embryonic organogenesis resulting from targeted disruption of the $\mathrm{N}$-myc gene in the mouse. Development 1993; 117: 1445-1455.

5 Kim J, Chu J, Shen X, Wang J, Orkin SH. An extended transcriptional network for pluripotency of embryonic stem cells. Cell 2008; 132: 1049-1061.

6 Kidder BL, Yang J, Palmer S. Stat3 and c-Myc genome-wide promoter occupancy in embryonic stem cells. PLoS One 2008; 3: e3932.

7 Kim J, Woo AJ, Chu J, Snow JW, Fujiwara Y, Kim CG et al. A Myc network accounts for similarities between embryonic stem and cancer cell transcription programs. Cell 2010; 143: 313-324.

8 Larsson LG, Henriksson MA. The Yin and Yang functions of the Myc oncoprotein in cancer development and as targets for therapy. Exp Cell Res 2010; 316: 1429-1437.

9 Li Z, Hann SR. The Myc-nucleophosmin-ARF network: a complex web unveiled. Cell Cycle 2009; 8: 2703-2707.

10 Cotter TG. Apoptosis and cancer: the genesis of a research field. Nat Rev Cancer 2009; 9: 501-507.

11 Shimada H, Ambros IM, Dehner LP, Hata J, Joshi VV, Roald B et al. The International Neuroblastoma Pathology Classification (the Shimada system). Cancer 1999; 86: 364-372.

12 Ying QL, Wray J, Nichols J, Batlle-Morera L, Doble B, Woodgett J et al. The ground state of embryonic stem cell self-renewal. Nature 2008; 453: 519-523.

13 Takahashi K, Yamanaka S. Induction of pluripotent stem cells from mouse embryonic and adult fibroblast cultures by defined factors. Cell 2006; 126: 663-676.

14 Silva J, Barrandon O, Nichols J, Kawaguchi J, Theunissen TW, Smith A. Promotion of reprogramming to ground state pluripotency by signal inhibition. PLOS Biol 2008; 6: e253.

15 Sridharan R, Tchieu J, Mason MJ, Yachechko R, Kuoy E, Horvath S et al. Role of the murine reprogramming factors in the induction of pluripotency. Cell 2009; 136: 364-377.

16 Mikkelsen TS, Hanna J, Zhang X, Ku M, Wernig M, Schorderet P et al. Dissecting direct reprogramming through integrative genomic analysis. Nature 2008; 454: 49-55.

17 Brodeur GM. Neuroblastoma: biological insights into a clinical enigma. Nat Rev Cancer 2003; 3: 203-216.

18 Nakagawara A. Neural crest development and neuroblastoma: the genetic and biological link. Prog Brain Res 2004; 146: 233-242.

19 Pardo M, Lang B, Yu L, Prosser H, Bradley A, Babu MM et al. An expanded Oct4 interaction network: implications for stem cell biology, development, and disease. Cell Stem Cell 2010; 6: 382-395. 
20 Varlakhanova NV, Cotterman RF, deVries WN, Morgan J, Donahue LR, Murray $S$ et al. myc maintains embryonic stem cell pluripotency and self-renewal. Differentiation 2010; 80: 9-19.

21 Smith KN, Singh AM, Dalton S. Myc represses primitive endoderm differentiation in pluripotent stem cells. Cell Stem Cell 2010; 7: 343-354.

22 Cartwright P, McLean C, Sheppard A, Rivett D, Jones K, Dalton S. LIF/STAT3 controls ES cell self-renewal and pluripotency by a Myc-dependent mechanism. Development 2005; 132: 885-896.

23 Maris JM, Denny CT. Focus on embryonal malignancies. Cancer Cell 2002; 2: 447-450.

24 Mosse YP, Laudenslager M, Longo L, Cole KA, Wood A, Attiyeh EF et al. Identification of ALK as a major familial neuroblastoma predisposition gene. Nature 2008; 455: 930-935.

25 Aiden AP, Rivera MN, Rheinbay E, Ku M, Coffman EJ, Truong TT et al. Wilms tumor chromatin profiles highlight stem cell properties and a renal developmenta network. Cell Stem Cell 2010; 6: 591-602.

26 Tada S, Era T, Furusawa C, Sakurai H, Nishikawa S, Kinoshita M et al. Characterization of mesendoderm: diverging point of the definitive endoderm and mesoderm in embryonic stem cell differentiation culture. Development 2005 132: 4363-4374.

27 Reynolds BA, Weiss S. Clonal and population analyses demonstrate that an EGF-responsive mammalian embryonic CNS precursor is a stem cell. Dev Biol 1996; 175: 1-13.

28 Ban H, Nishishita N, Fusaki N, Tabata T, Saeki K, Shikamura M et al. Efficient generation of transgene-free human induced pluripotent stem cells (iPSCs) by temperature-sensitive Sendai virus vectors. Proc Natl Acad Sci USA 2011; 108: 14234-14239.

29 Wartiovaara K, Barnabe-Heider F, Miller FD, Kaplan DR. Mycn promotes survival and induces S-phase entry of postmitotic sympathetic neurons. J Neurosci 2002; 22: $815-824$.

30 Nishishita N, ljiri H, Takenaka C, Kobayashi K, Goto K, Kotani E et al. The use of leukemia inhibitory factor immobilized on virus-derived polyhedra to support the proliferation of mouse embryonic and induced pluripotent stem cells. Biomaterials 2011; 32: 3555-3563.

31 Yoshimizu T, Sugiyama N, De Felice M, Yeom Yl, Ohbo K, Masuko K et al. Germline-specific expression of the Oct-4/green fluorescent protein (GFP) transgene in mice. Dev Growth Differ 1999; 41: 675-684.

32 Miyoshi $\mathrm{H}$. Gene delivery to hematopoietic stem cells using lentiviral vectors. Methods Mol Biol 2004; 246: 429-438.

33 Guo G, Yang J, Nichols J, Hall JS, Eyres I, Mansfield W et al. Klf4 reverts developmentally programmed restriction of ground state pluripotency. Development 2009; 136: 1063-1069.

34 Tachibana M, Matsumura Y, Fukuda M, Kimura H, Shinkai Y. G9a/GLP complexes independently mediate $\mathrm{H} 3 \mathrm{~K} 9$ and DNA methylation to silence transcription. $E M B O$ J 2008; 27: 2681-2690

(c) This work is licensed under the Creative Commons AttributionNonCommercial-No Derivative Works 3.0 Unported License. To view copy of this license, visit http://creativecommons.org/licenses/by-nc-nd/3.0/

Supplementary Information accompanies the paper on the Oncogene website (http://www.nature.com/onc) 